\title{
Docosahexaenoic acid inhibits vascular smooth muscle cell migration and proliferation by decreasing microRNA-155 expression levels
}

\author{
XIAOLIANG YIN, CHUNBO XU, QIYANG XU and DEHAI LANG
}

Department of Vascular Surgery, Hwa Mei Hospital, University of Chinese Academy of Sciences (Ningbo No. 2 Hospital), Ningbo Institute of Life and Health Industry, University of Chinese Academy of Sciences, Ningbo, Zhejiang 315010, P.R. China

Received June 17, 2018; Accepted June 12, 2020

DOI: $10.3892 / \mathrm{mmr} .2020 .11404$

\begin{abstract}
Vascular smooth muscle cell (VSMC) hyperplasia is a common cause of carotid restenosis. In the present study, the potential protective effects of docosahexaenoic acid (DHA) in carotid restenosis and the underlying mechanism of its effects were examined. VSMCs were treated with DHA, a polyunsaturated $\omega-3$ fatty acid. Cell migration and proliferation were assessed using wound healing and Cell Counting Kit-8 assays and by measuring Ki- 67 protein levels. Additionally, the expression levels of microRNA-155 were determined by reverse transcription-quantitative PCR (RT-qPCR). The involvement of microRNA-155 in the regulation of migration and proliferation was evaluated by transfecting VSMCs with microRNA mimics and inhibitors. Moreover, the reversal of migration and proliferation after transfection of VSMCs with the microRNA mimics and subsequent treatment with DHA was investigated. A target gene of microRNA-155 was identified using RT-qPCR and luciferase assays. The migration and proliferation of VSMCs, as well as the expression of microRNA-155 was inhibited by DHA stimulation. MicroRNA-155 regulated the migration and proliferation of VSMCs. Finally, proliferation and migration of VSMCs were reduced following DHA treatment, which was mediated by an increase in the expression levels of microRNA-155. Suppressor of cytokine signalling 1 (Socs1) was the target gene of microRNA-155. In conclusion, DHA inhibited VSMC migration and proliferation by reducing microRNA-155 expression. This effect may be caused by the microRNA-155 target gene Socs1.
\end{abstract}

Correspondence to: Dr Dehai Lang, Department of Vascular Surgery, Hwa Mei Hospital, University of Chinese Academy of Sciences (Ningbo No. 2 Hospital), Ningbo Institute of Life and Health Industry, University of Chinese Academy of Sciences, 41 Xibei Street, Ningbo, Zhejiang 315010, P.R. China

E-mail: byeyyu@163.com

Key words: docosahexaenoic acid, microRNA-155, suppressor of cytokine signalling 1 , vascular smooth muscle cells

\section{Introduction}

Carotid restenosis has been associated with an increased risk of ischaemic stroke and with increasing morbidity and mortality $(1,2)$. Studies investigating the pathogenesis of carotid restenosis are vital for the development of novel prevention and treatment strategies. Abnormal proliferation and migration of vascular smooth muscle cells (VSMCs) is a common pathogenetic factor in carotid artery stenosis. Inhibition of the hypertrophy of VSMCs might be an effective method for treatment of carotid artery stenosis.

Docosahexaenoic acid (DHA) is an important component of fish oil, which is generally used in the prevention of cardiovascular disease (3). A number of previous studies demonstrated the anti-inflammatory and lipid-regulating effects of DHA in cardiovascular disease $(4,5)$. The mechanism underlying the DHA protective effect in cardiovascular diseases requires additional investigation. DHA has been demonstrated to regulate the VSMC cycle by stimulating apoptosis, and by inhibiting proliferation and migration $(6,7)$. In another previous study, the beneficial effect of DHA on the migration of VSMCs was partially dependent on the regulation of matrix metalloproteinase (MMP)-2 and MMP-9 (8). Moreover, DHA could increase nitric oxide (NO) production through activation of the p44/p42/MAPK signalling in VSMCs (6). Another mechanism of the protective effect of DHA involves regulation of VSMC proliferation through the inhibition of phosphorylation of the cyclin-dependent kinase 2/cyclin E complex (9). However, the mechanism underlying the inhibitory effect of DHA on the proliferation and migration of VSMCs requires further investigation.

The protective mechanism of DHA in vascular pathology has been partially elucidated, which suggested that biological changes were predominantly caused by an abnormal expression of genes involved in proliferation, migration, cell cycle and apoptosis (10-13). Moreover, several previous studies have reported that non-coding RNAs, including microRNAs, regulate these biological processes. For instance, microRNA-155 is a multifunctional non-coding RNA which regulates the pathological processes involved in cardiovascular disease (14). Indeed, microRNA-155 participates in the regulation of haematopoietic lineage differentiation, vascular remodelling, viral infection and inflammation (15-18). Genetic deficiency of 
microRNA-155 in cardiomyocytes prevents cardiac hypertrophy and failure (19). Furthermore, mircroRNA-155 has been shown to act as a tumour suppressor and an oncogene in a various cancer types $(20,21)$. MicroRNA-155 can regulate glucose usage through the PIK3R1-PDK/AKT-FOXO3a-cMYC axis, according to a previous study on 50 triple-negative breast cancer specimens (22). However, it is unclear whether microRNA-155 can modulate the VSMC phenotype, or participate in the protective effect of DHA in carotid restenosis.

In the present study, DHA had an inhibitory effect on the proliferation and migration of VSMCs, which was mediated by regulation of miR-155 expression. The present results could be applied in the clinical prevention of carotid restenosis. In addition, the present study identified a positive effect of microRNA-155 on the proliferation and migration of VSMCs, in agreement with previous studies $(23,24)$. The phenotype changes of the DHA-stimulated VSMCs were reversed by inhibiting the expression of microRNA-155. Overall, these effects may involve regulation of the miR-155 target gene suppressor of cytokine signalling 1 (Socs1).

\section{Materials and methods}

Cell culture. VSMCs were isolated from the aorta of 8-week-old male Wistar rats (180-270 g) (Vital River), according to a previously published protocol (8). The Ethics Committee of Hwa Mei Hospital, University of Chinese Academy of Sciences (Ningbo No. 2 Hospital) approved the present study. Rats were housed at $20-25^{\circ} \mathrm{C}$ with a $12 \mathrm{~h}$ light/dark cycle and free access to food and water. Briefly, adventitia was quickly removed from a sacrificed rat for enzymatic digestion after removing the fat tissue and mechanically extruding the media. Cells were cultured in DMEM, supplemented with 10\% FBS (both Gibco; Thermo Fisher Scientific, Inc.) and $100 \mathrm{U} / \mathrm{ml}$ penicillin and $0.1 \mathrm{mg} / \mathrm{ml}$ streptomycin antibiotics. All cultured cells were maintained at $37^{\circ} \mathrm{C}$ in a humidified $5 \% \mathrm{CO}_{2}$ incubator. The cells were passaged 3-7 times before performing the experiments. The cell type and the maintenance of the differentiated state were confirmed by the presence of a well-structured network of actin stress fibres, according to the immunochemistry data (data not shown).

MicroRNA transfection. The microRNA-155 mimics, mimics negative control, microRNA-155 inhibitors and inhibitor negative control were obtained from Guangzhou RiboBio Co., Ltd. (sequences not available). VSMCs were seeded into 6-well cell culture plates at density of $5 \times 10^{6}$ cells per well prior to transfection. Following a 20-h incubation, cells were transfected with miRNA at final concentration of $50 \mathrm{nM}$ using Lipofectamine ${ }^{\circledR}$ 3000 (Invitrogen; Thermo Fisher Scientific, Inc.) according to the manufacturer's instructions. After a 6-h incubation with miRNAs, the medium was changed to fresh complete medium. Transfected cells were grown for $24 \mathrm{~h}$ prior to subsequent experiments.

$R N A$ isolation and reverse transcription-quantitative $P C R$ (RT-qPCR). VSMCs were treated with $50 \mu \mathrm{M}$ DHA (Sigma-Aldrich; Merck KGaA) for $2 \mathrm{~h}$, then cells were transfected with microRNA-155 mimics and inhibitors before harvesting for RNA isolation. VSMCs were centrifuged at $4^{\circ} \mathrm{C}$ with $700 \mathrm{x} g$ for $5 \mathrm{~min}$ for harvesting after cells were transfected for 24 h. RNA was extracted using TRIzol ${ }^{\circledR}$ (Invitrogen; Thermo Fisher Scientific, Inc.), according to the manufacturer's protocol. The concentration, purity and integrity of the RNA were measured using an ultraviolet spectrophotometer, as well as $0.8 \%$ agarose gel electrophoresis. Reverse transcription was performed using an RT kit (MicroRNA Reverse Transcription kit; Applied Biosystems; Thermo Fisher Scientific, Inc.) with $1 \mu \mathrm{g}$ total RNA. The RT conditions were as follows: $25^{\circ} \mathrm{C}$ for $10 \mathrm{~min}, 37^{\circ} \mathrm{C}$ for $120 \mathrm{~min}$, and $85^{\circ} \mathrm{C}$ for $5 \mathrm{~min}$. The PCR primer sequences for miR-155 were: Forward, 5'-CTGTTAATGCTA ATCGTGATAG-3' and reverse, 5'-GCAGGGTCCGAGGT-3'. The PCR primer sequences for Socs1 were 5'-CTGCGGCTT CTATTGGGGAC-3' and 5'-AAAAGGCAGTCGAAGGTC TCG-3'. The PCR primers of snoRNA U6 (internal control) were: Forward, 5'-GCGCGTCGTGAAGCGTTC-3' and reverse, 5'-GTGCAGGGTCCGAGGT-3'. The PCR primers of GAPDH (internal control) were: Forward, 5'-CGGAGTCAA CGGATTTGGTCGTAT-3' and reverse, 5'-AGCCTTCTCCAT GGTGGTGAAGAC-3'. The fluorophore was SYBR Green (Roche Applied Science). The real-time PCR conditions were as follows: $95^{\circ} \mathrm{C}$ for $10 \mathrm{~min}, 60^{\circ} \mathrm{C}$ for $10 \mathrm{sec}, 72^{\circ} \mathrm{C}$ for $30 \mathrm{sec}$ (40 cycles) and $4^{\circ} \mathrm{C}$ for $5 \mathrm{~min}$. The real-time PCR amplification scheme was performed according to the manufacturer's instructions (Roche Applied Science). The concentration of microRNAs were calculated by the $2^{-\Delta \Delta \mathrm{Cq}}$ method (25).

Cell viability. After the VSMCs were transfected with the miR-155 mimics and inhibitors, the VSCMs were grown in 96-well cell culture plates at $5 \times 10^{5}$ cells per well. The VSMCs were then harvested and kept in the same medium for the detection of proliferation, following standard culture for 24, 48 or $72 \mathrm{~h}$. Cell proliferation was assessed using a Cell Counting Kit-8 (CCK-8) assay (Abcam). All procedures were performed according to the manufacturer's protocol. Absorbance was detected using a microplate reader at $450 \mathrm{~nm}$.

Wound healing assay. VSMCs were treated with $50 \mu \mathrm{M}$ DHA and transfected with microRNA-155 mimics and inhibitors before evaluating their migration in the wound heal assay. After transfection for $6 \mathrm{~h}$, a sterile $10-\mu \mathrm{l}$ white tip was used to draw a straight line across the $3.5 \mathrm{~cm}$ dish, with the tip on the bottom of the plate, and the growth medium was replaced with serum-free medium. At this time point, $0 \mathrm{~h}$, images were recorded using a light microscope (magnification, x40). Subsequently, microscopic images were recorded at 24 and $48 \mathrm{~h}$ after wounding.

Luciferase reporter assay. Socs1, the target gene of microRNA-155, was predicted by inputting the microRNA sequence in TargetScan web (targetscan.org/). The Socs1-3' untranslated region (UTR) DNA fragment, including the putative microRNA-155 binding sequence, was cloned into the PGL3-basic (Promega Corporation) (hereafter referred to as PGL3/Socs1-3'UTR). Additionally, a mutant Socs1-3'UTR plasmid was constructed by mutating the miR-155 binding sequence (referred to as PGL3/Socs1-3'UTR mutant thereafter). VSMCs were plated in a 24 -well plate at $1 \times 10^{5}$ cells per well before transfection using Lipofectamine 3000) (Thermo Fisher Scientific, Inc.). Each transfection contained the vector DNA and pRL-SV40 plasmid. The pGL3-basic vector was used as a negative control. PRL-TK (Promega Corporation) was used 
A

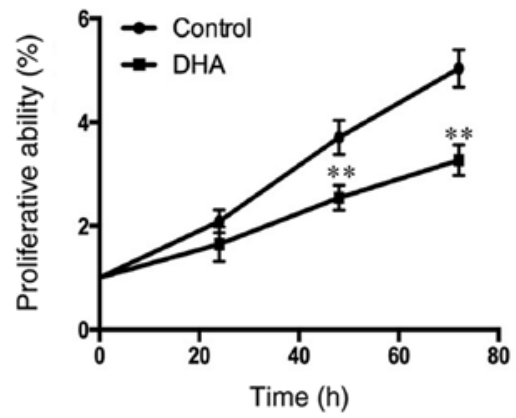

C

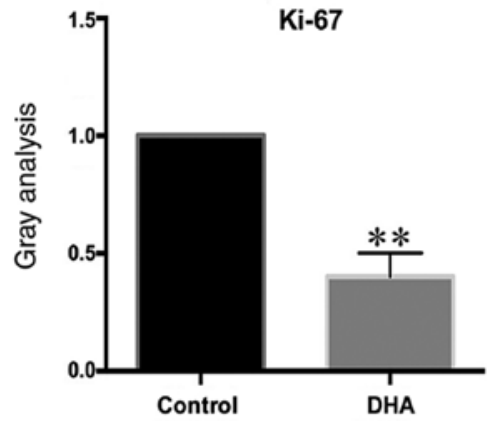

E

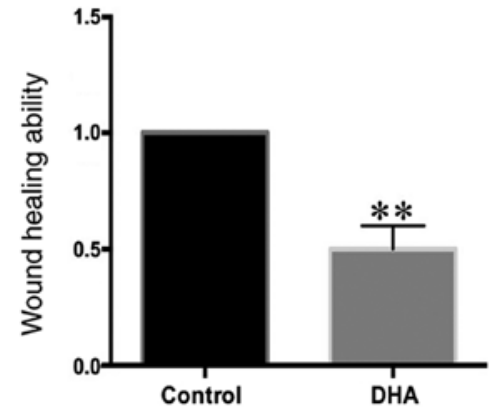

B

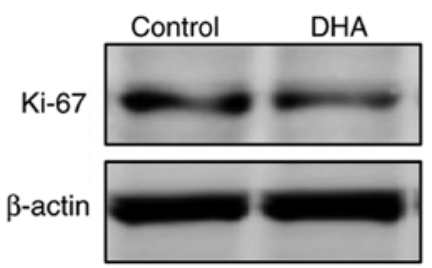

D

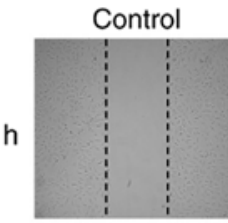

DHA

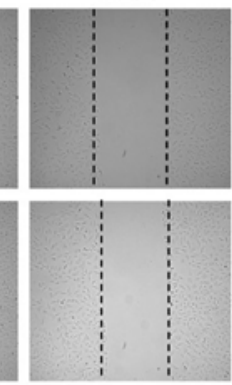

$\mathrm{F}$

$24 \mathrm{~h}$

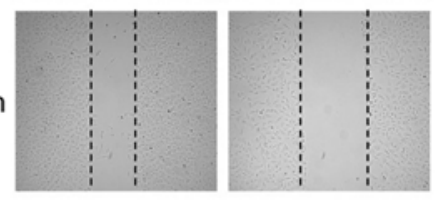

MicroRNA-155

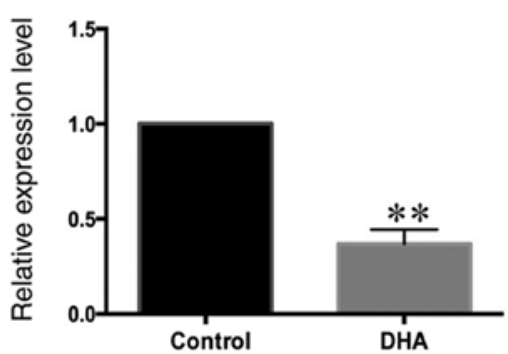

Figure 1. DHA inhibits the proliferation and migration of VSMCs. VSMCs were treated with $50 \mu \mathrm{M}$ DHA then harvested for the assessment of proliferation and migration. (A) Proliferation of VSMCs treated with DHA was evaluated using a Cell Counting Kit-8 assay. (B) Proliferation of DHA-treated VSMCs was evaluated by the Ki-67 protein level. (C) Densitometry analysis of the relative protein levels of Ki-67. (D) Migration of VSMCs treated with DHA was evaluated using a wound healing assay (magnification, x100). (E) Statistical analysis of migration of VSMCs treated with DHA. (F) Relative expression level of microRNA-155 was analysed by reverse transcription-quantitative PCR. ${ }^{* *} \mathrm{P}<0.01$ vs. respective control. VSMCs, vascular smooth muscle cells; DHA, docosahexaenoic acid.

as the internal control. Luciferase assays were performed $48 \mathrm{~h}$ after transfection, and independent experiments were repeated three times for each plasmid construct according to the manufacturer's protocol (Promega Corporation). The luciferase activity was measured via fluorescence spectrophotometer (Luminex Corporation). The ratio between luciferase activity and Renilla luciferase activity represented the value of measured sample.

Western blotting. VSMCs were treated with $50 \mu \mathrm{M}$ DHA and transfected with the microRNA-155 mimics and inhibitors before harvesting for protein isolation. Proteins were isolated in the protein extraction buffer (Beyotime Institute of Biotechnology). The protein concentration was measured by BCA method. Protein samples $(20 \mu \mathrm{g} / \mathrm{lane})$ were separated by SDS-PAGE on $10 \%$ gels and transferred to a nitrocellulose membrane. The membranes were incubated with 5\% fat-free milk in TBS with Tween $^{\circledR} 20$ for $1 \mathrm{~h}$ at room temperature and with primary antibodies overnight at $4^{\circ} \mathrm{C}$. The PVDF membranes were washed with TBST buffer and incubated with peroxidase-conjugated specific secondary antibodies from Abcam [Ki67 (1:5,000; cat. no. ab15580), Socs1 (1:4,000; cat. no. ab62584) and actin
(1:3,000; cat. no. ab6276)] with shaking for $1 \mathrm{~h}$. The enhanced chemiluminescence solution (Sigma-Aldrich; Merck KGaA) was the prepared in a dark room, and the exposure time of the film was determined according to the chemiluminescence intensity. Densitometric analysis was performed using Image Lab software (version 3.0; Bio-Rad Laboratories, Inc.).

Statistical analysis. Results are presented as the mean \pm standard deviation of three replicate experiments. For multiple comparisons, statistical analysis was conducted using ANOVA, followed by Sidak's post hoc test. A Student's t-test was used for statistical analysis of two groups. All the experiments were repeated at least for three times. $\mathrm{P}<0.05$ was considered to indicate a statistically significant difference. IBM SPSS Statistics software (version 21.0; IBM Corp.) was used to perform all the statistical analyses.

\section{Results}

DHA inhibits the proliferation and migration of VSMCs. VSMCs were stimulated with $50 \mu \mathrm{M}$ DHA to evaluate its effects on proliferation and migration. VSMC proliferation following DHA 

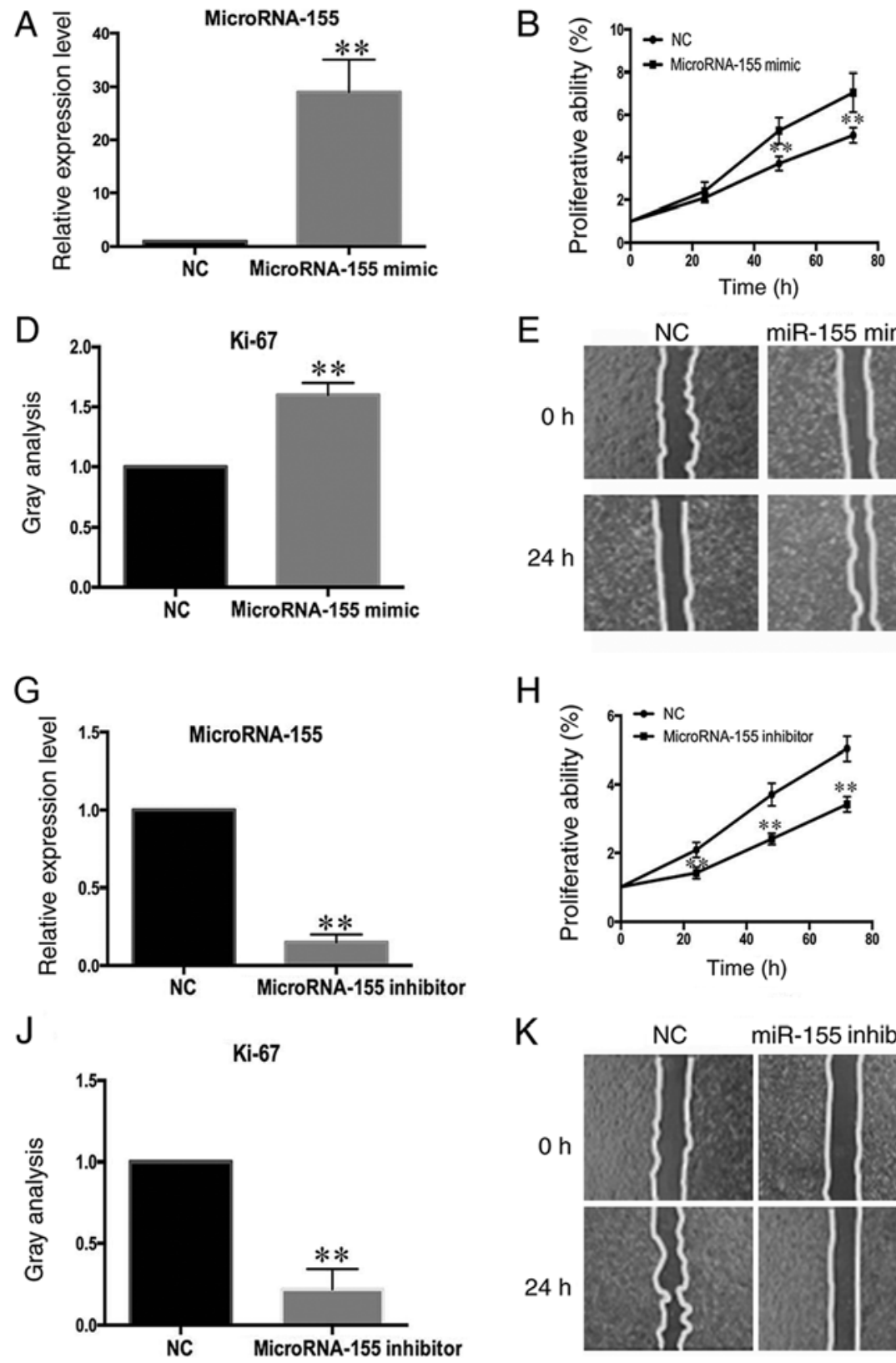

E

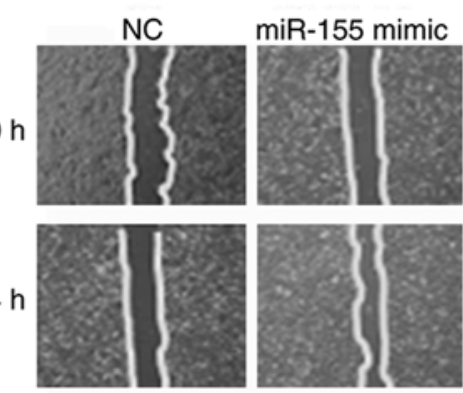

$\mathrm{H}$

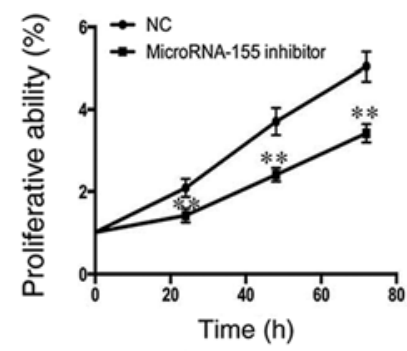

$\mathrm{K}$

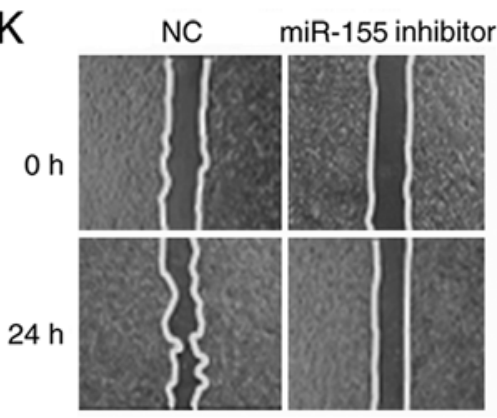

C

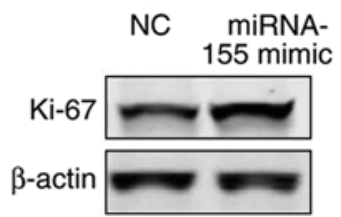

$\mathrm{F}$

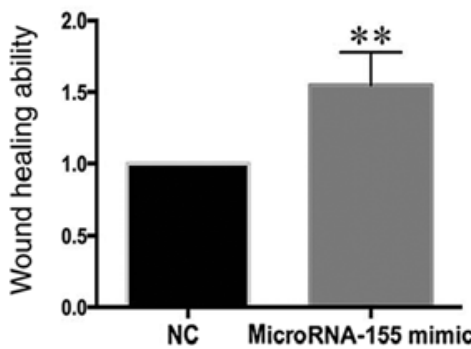

I

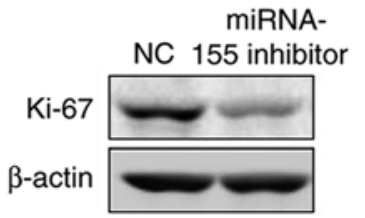

$\mathrm{L}$

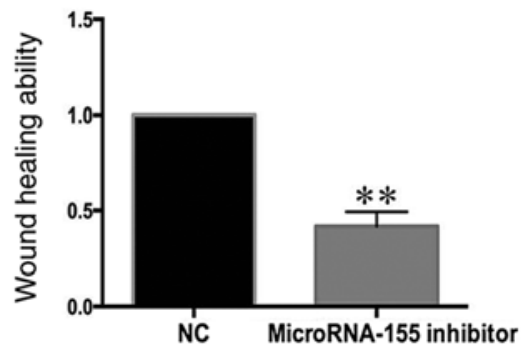

Figure 2. MicroRNA-155 regulates the proliferation and migration of VSMCs. VSMCs were transfected with microRNA-155 mimics and inhibitors. (A) Efficiency of miR-155-mimics transfection was evaluated by RT-qPCR. (B) Proliferation of miR-155-mimics-transfected cells was evaluated using a CCK-8 assay. (C) Proliferation of miR-155 mimics-transfected cells was evaluated by measuring Ki-67 protein levels. (D) Densitometry analysis of the relative protein levels of Ki-67. (E) Migration of VSMCs transfected with miR-155-mimic was evaluated using a wound healing assay (magnification. x100). (F) Statistical analysis of the migration potential of VSMCs transfected with miR-155-mimic. (G) Effect of miR-155-inhibitor was evaluated by RT-qPCR. (H) Proliferation of miR-155 inhibitor-transfected cells was evaluated using a CCK-8 assay. (I) Proliferation of miR-155-inhibitor-transfected cells was evaluated by measuring Ki-67 protein levels. (J) Densitometry analysis of the relative protein levels of Ki-67. (K) Migration of VSMCs transfected with miR-155-inhibitor was evaluated using a wound healing assay. (L) Statistical analysis of the migration potential of VSMCs transfected with miR-155-inhibitor. ${ }^{* *} \mathrm{P}<0.01$, vs. NC. VSMCs, vascular smooth muscle cells; DHA, docosahexaenoic acid; miR/miRNA, microRNA; NC, negative control; RT-qPCR, reverse transcription-quantitative PCR; CCK-8, Cell Counting Kit-8.

treatment was evaluated using the CCK-8 assay and by measuring the Ki-67 protein levels (Fig. 1A and B). The proliferation of VSMCs treated with DHA for $48 \mathrm{~h}$ was significantly decreased, compared with untreated cells (Fig. 1A). The effect of DHA on VSMC migration was then assessed, using a wound healing assay. The migration of VSMCs treated with DHA was also decreased, compared with untreated cells (Fig. 1C-E). immune response and development of T cells $(26,27)$. In order to determine whether microRNA-155 might participate in the regulation of the biological activities of VSMCs, the expression levels of microRNA-155 in DHA-stimulated VSMCs were measured using RT-qPCR. The expression levels of microRNA-155 were significantly decreased in DHA-treated cells, compared with untreated cells (Fig. 1F).

MicroRNA-155 regulates the proliferation and migration of VSMCs. The role of miR-155 in the regulation of VSMC 
A

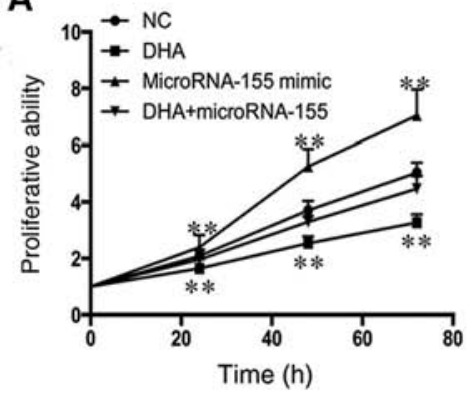

B

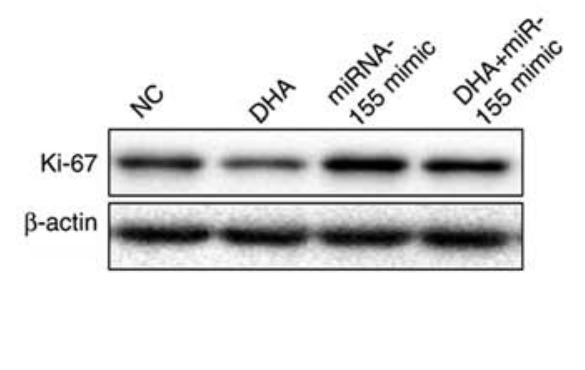

C

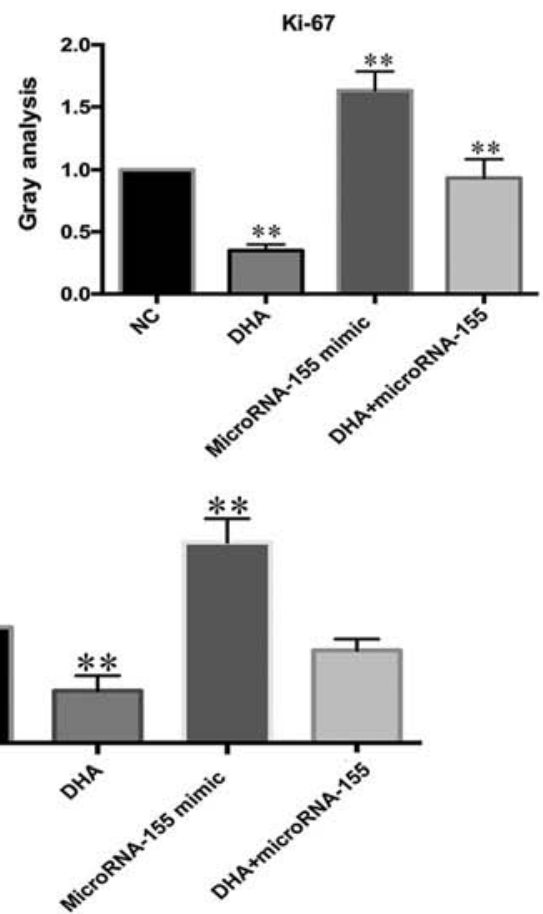

Figure 3. Restoration of the expression levels of microRNA-155 partially reverses the inhibition of VSMC proliferation and migration. VSMCs were transfected with miR-155-mimic before treatment with $50 \mu \mathrm{M}$ DHA. Cells were divided into four groups: NC, DHA, microRNA-155-mimic and DHA + microRNA-155-mimic group. (A) Proliferation in each group was evaluated using a Cell Count Kit-8 assay. (B) Proliferation of each group was evaluated by measuring Ki-67 protein levels. (C) Densitometry analysis of the relative protein levels of Ki-67. (D) Migration of each group of cells was evaluated using a wound healing assay (magnification, $\mathrm{x} 100$ ). (E) Statistical analysis of the migration potential in each group. ${ }^{* * *} \mathrm{P}<0.01 \mathrm{vs.} \mathrm{NC}$. VSMCs, vascular smooth muscle cells; DHA, docosahexaenoic acid; NC, negative control.

proliferation and migration was also investigated. The proliferation of VSMCs transfected with miR-155 mimic (Fig. 2A) and microRNA-155-inhibitor (Fig. 2G) was evaluated using a CCK-8 assay and by measuring the protein levels of Ki-67. The proliferation of VSMCs was increased by the microRNA-155 mimic (Fig. 2B-D). Conversely, the proliferation of VSMCs transfected with the microRNA-155 inhibitor was significantly decreased (Fig. 2H-J). These results demonstrated that microRNA-155 can regulate the proliferation of VSMCs. The potential effect of microRNA-155 on VSMC migration was then assessed. After confirming the efficiency of overexpression and interference by RT-qPCR (Fig. 2A and G), the migration of VSMCs was evaluated using a wound healing assay. The migration of VSMCs was increased following microRNA-155 overexpression (Fig. 2E and F) and was decreased following transfection of microRNA-155-inhibitor (Fig. 2K and L). These results suggested that microRNA-155 can regulate the migration potential of VSMCs.

Restoration of the expression level of microRNA-155 partially reverses the inhibition of VSMC proliferation and migration. MicroRNA-155 plays an essential role in the regulation of VSMC proliferation and migration (7). In order to investigate whether a decrease in proliferation and migration in the DHA-stimulated VSMCs was caused by microRNA-155 downregulation, VSMCs were transfected with microRNA-155 mimic, then treated with DHA $(50 \mu \mathrm{M})$. The proliferation of VSMCs was analysed using a CCK- 8 assay and measurement of Ki-67 protein levels. The inhibition of VSMC proliferation was partially reversed by microRNA- 155 overexpression (Fig. 3A-C). In agreement with this, the migration of DHA-stimulated VSMCs was also reversed according to the wound healing assay (Fig. 3D and E). Thus, overexpression of microRNA-155 restored the proliferation and migration of DHA-stimulated VSMCs.

Socs1 is the target gene of microRNA-155 in VSMCs. Identification of the target gene of microRNA-155 is important for understanding the biological functions of microRNA-155, and is required to investigate a possible mechanism of the regulatory effects of microRNA-155 on VSMC proliferation and migration. According to the target gene prediction data generated by TargetScan (targetscan.org/), Socs1 was a candidate target based on a study in macrophages (28). Socs1 is a suppressor of cytokine signalling, which is required for cell growth and survival (28). The mRNA levels of Socs1 were significantly increased following transfection with the microRNA-155-mimics, compared with the control (Fig. 4A). By contrast, the expression of Socs1 was increased in the absence of microRNA-155 (Fig. 4B). In order to find direct evidence for the interaction between Socs1 and microRNA-155, a luciferase plasmid containing the Socs1 gene 3'UTR (PGL3/Socs1-3'UTR) was constructed. Transfection of the microRNA-155-mimic significantly suppressed the luciferase activity of PGL3/Socs1-3'UTR (Fig. 4C) but had no effect on the PGL3/Socs1-3'UTR mutant plasmid (Fig. 4D), suggesting that Socs1 is the direct target gene of microRNA-155. In order to demonstrate that Socs1 is regulated by DHA through microRNA-155, the expression levels of Socs1 were measured in 
A

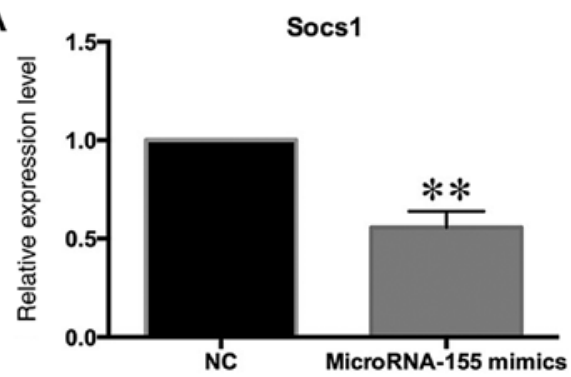

C

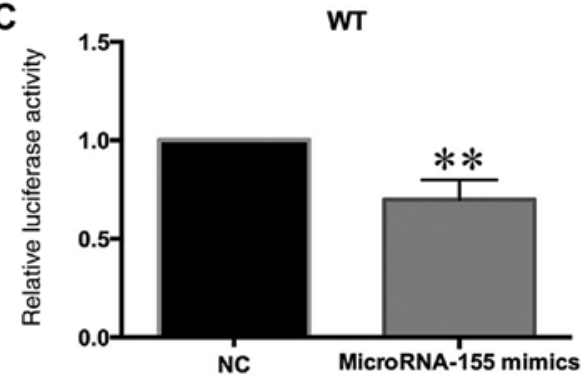

E

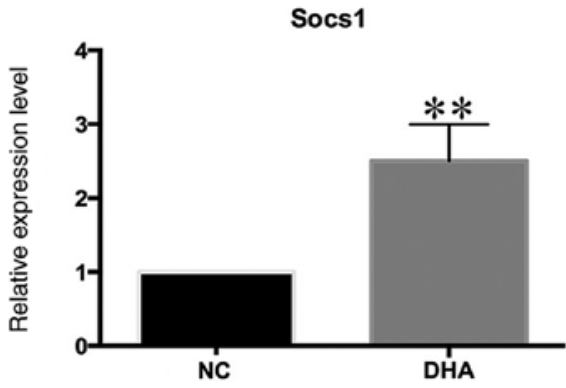

G

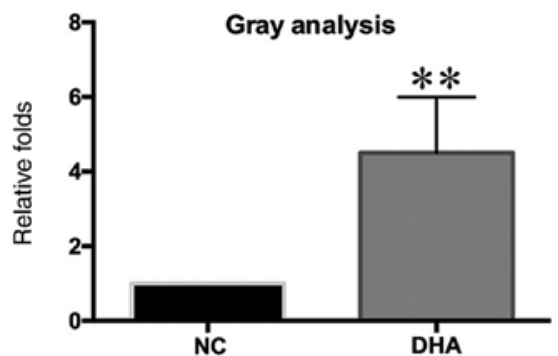

B

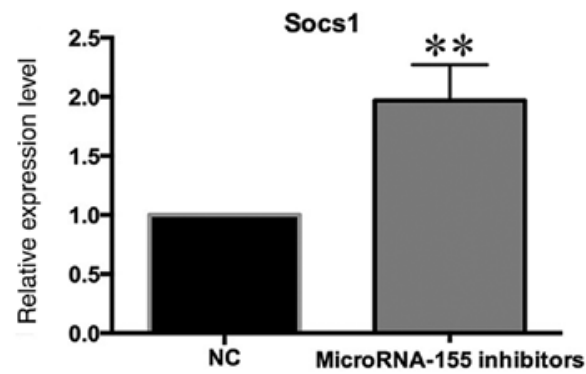

D

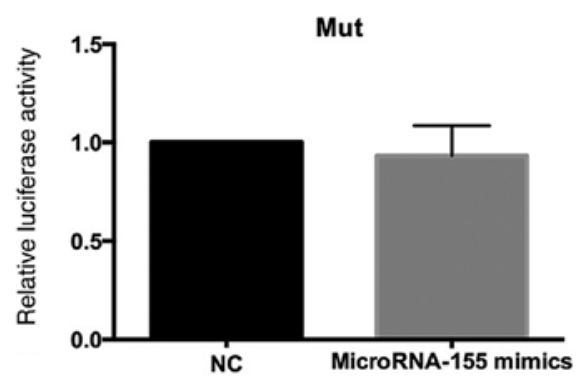

$\mathbf{F}$

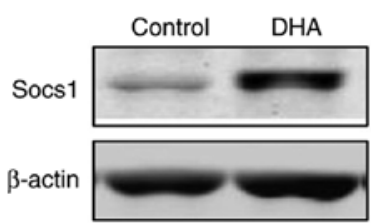

Figure 4. Socs1 is the target gene of microRNA-155. VSMCs were transfected with miR-155-mimic and inhibitor. Expression levels of Socs1 was analysed by RT-qPCR. (A) Expression level of Socs1 was decreased in miR-155-mimic-transfected cells, vs. NC. (B) Expression level of Socs1 was increased in miR-155-inhibitor-transfected cells, compared with NC. (C) Luciferase plasmid WT PGL3/Socs1-3'UTR was transfected, together with miR-155-mimic. Luciferase activity of WT PGL3/Socs1-3'UTR was significantly decreased. (D) Luciferase plasmid Mut PGL3/Socs1-3'UTR was transfected, together with miR-155-mimic. Luciferase activity of Mut PGL3/Socs1-3'UTR remained unchanged. VSMCs were treated with $50 \mu \mathrm{M}$ DHA, and Socs1 expression levels were analysed by RT-qPCR and western blotting. (E) Expression levels of Socs1 were increased in the DHA-stimulated VSMCs, compared with NC. (F) Protein levels of Socs1 were increased in the DHA-stimulated VSMCs, compared with NC. (G) Gray analysis of the relative protein levels of Socs1. ${ }^{* *} \mathrm{P}<0.01$ compared with NC. VSMCs, vascular smooth muscle cells; DHA, docosahexaenoic acid; NC, negative control; RT-qPCR, reverse transcription-quantitative PCR; WT, wild-type; Mut, mutant; miR, microRNA; Socs1, suppressor of cytokine signalling.

DHA-stimulated VSMCs. Both the protein and mRNA levels of Socs1 were increased following DHA stimulation (Fig. 4E-G).

\section{Discussion}

In the present study, the protective mechanism of DHA in the inhibition of VSMC proliferation and migration was investigated. Previous studies on the protective effect of DHA in cardiovascular diseases focused on changes in gene expression $(29,30)$. The present study demonstrated that the inhibitory effect of DHA on the proliferation and migration of VSMCs is mediated by microRNA-155 downregulation. MicroRNA-155 is a multifunctional non-coding RNA that plays an important role in the pathogenesis of carotid restenosis (31). However, the Socs1 target gene of microRNA-155 can regulate various signalling pathways, which may contribute to the function of 
microRNA-155 in the regulation of VSMC proliferation and migration.

A recent study demonstrated that low DHA levels were significantly associated with lipid-rich coronary and carotid plaques, yet the mechanism of DHA in the stabilization of carotid plaque and its beneficial effects in patients with carotid restenosis require additional investigation (32). DHA is an important component of $\omega-3$ fatty acids, which can enhance Gefitinib sensitivity and induce apoptosis in PC-9/GR cells (33). According to nutritional statistical data, low levels of $\omega-3$ fatty acids are associated with cerebral small vessel diseases, hypertension, cardiovascular dysfunction and acute ischaemic stroke $(34,35)$. The present study focused on the protective function of DHA in carotid restenosis by evaluating its effect on VSMC hyperplasia through the regulation of proliferation and migration.

MicroRNA-155 is one of the most multifunctional non-coding RNAs, and it participates in the regulation of numerous biological functions in various cell types, including cells of the immune system (36). In the present study, microRNA-155 regulated the proliferation and migration of VSMCs, possibly through its target gene, Socs1. As a multifunctional microRNA, microRNA-155 could also participate in other biological processes. Numerous previous studies have been published on the function of microRNA-155 and the mechanisms underlying its effects (36-38). MicroRNA-155 could inhibit apoptosis by regulating PTEN signalling in psoriasis (37). The function of microRNA-155 in cardiac hypertrophy was demonstrated in a mouse model, suggesting that loss of microRNA-155 expression might prevent the progression of heart failure (38). In cancer cells, microRNA-155 deficiency can prevent tumour growth by enhancing the function and recruitment of tumour suppressor cells in the tumour micro-environment (39). The function of microRNA-155 is mediated by regulation of a series of signalling pathways. In fibrosis, microRNA-155 was strongly associated with the activation of $\mathrm{Wnt} / \beta$-catenin signalling (40). Moreover, a previous study demonstrated that the immunoregulatory function of microRNA-155 was mediated by the negative regulation of Akt and Stat5 signalling (41). Socs1 is not the only known target gene of microRNA-155 (42); various signalling pathways regulated by microRNA-155 may contribute to the phenotype of DHA-stimulated VSMCs. The present study predominantly focused on the mechanism of microRNA-155 regulation of cell proliferation and migration.

Socs1 is an important target gene of microRNA-155 according to numerous previous studies $(43,44)$. However, its functions in microvascular endothelial cells and VSMCs remain poorly studied. Several previous studies focused on the regulatory role of Socs1 in the immune system; for instance, Socs1 was demonstrated to be involved in the inflammatory response in atherogenesis $(45,46)$. In cancer cells, the Socs1 gene acts a tumour suppressor and is frequently silenced by hypermethylation of the promoter (47). Socs1 tumour suppressor activity is mediated by enhancing p53 tumour suppressor activity and by inhibiting the Met receptor (47). In the present study, Socs1 expression levels were increased in DHA-stimulated VSMCs, suggesting that it may serve a role in the proliferation and migration of VSMCs. However, the underlying mechanism requires further investigation, and the function of Socs1 in VSMCs still requires extensive evaluation.

In conclusion, the present study demonstrated that DHA could inhibit the proliferation and migration of VSMCs, which may be a possible mechanism for the protective effect of DHA in carotid restenosis. To the best of our knowledge, the present study is the first to indicate that changes in microRNA expression may influence the activity of VSMCs. The function of microRNA-155 might be varied in different cell types. Further studies and experiments are required, and should focus on functional studies of microRNA-155 in vascular disease.

\section{Acknowledgements}

Not applicable.

\section{Funding}

The present study was supported by Ningbo Health Branding Subject Fund (grant no. PPXK2018-01), Ningbo Natural Science Fund (grant no. 2019A610345) and Hwamei Scientific Research Fund (grant no. 2018HMKY56).

\section{Availability of data and materials}

The datasets used and/or analyzed during the current study are available from the corresponding author on reasonable request.

\section{Authors' contributions}

DL and XY designed and initiated the present study. XY and CX carried out the cell culture experiments. QX performed RT-qPCR analysis in cultured cells. DL and XY wrote the manuscript. All authors read and approved the final manuscript.

\section{Ethics approval and consent to participate}

The present study was approved by The Ethics Committee of Hwa Mei Hospital, University of Chinese Academy of Sciences (Ningbo No. 2 Hospital).

\section{Patient consent for publication}

Not applicable.

\section{Competing interests}

The authors declare that they have no competing interests.

\section{References}

1. Bonati LH, Ederle J, Dobson J, Engelter S, Featherstone RL, Gaines PA, Beard JD, Venables GS, Markus HS, Clifton A, et al: Length of carotid stenosis predicts peri-procedural stroke or death and restenosis in patients randomized to endovascular treatment or endarterectomy. Int J Stroke 9: 297-305, 2014.

2. Plummer C, Henderson RD, O'Sullivan JD and Read SJ: Ischemic stroke and transient ischemic attack after head and neck radiotherapy: A review. Stroke 42: 2410-2418, 2011.

3. Schunck WH, Konkel A, Fischer R and Weylandt KH: Therapeutic potential of omega-3 fatty acid-derived epoxyeicosanoids in cardiovascular and inflammatory diseases. Pharmacol Ther 183: 177-204, 2018. 
4. Iverson C, Bacong A, Liu S, Baumgartner S, Lundstrom T, Oscarsson J and Miner JN: Omega-3-carboxylic acids provide efficacious anti-inflammatory activity in models of crystal-mediated inflammation. Sci Rep 8: 1217, 2018.

5. Trattner S, Ruyter B, Ostbye TK, Kamal-Eldin A, Moazzami A, Pan J, Gjoen T, Brännäs E, Zlabek V and Pickova J: Influence of dietary sesamin, a bioactive compound on fatty acids and expression of some lipid regulating genes in Baltic Atlantic salmon (Salmo salar L.) juveniles. Physiol Res 60: 125-137, 2011.

6. Hirafuji M, Machida T, Tsunoda M, Miyamoto A and Minami M: Docosahexaenoic acid potentiates interleukin-lbeta induction of nitric oxide synthase through mechanism involving p44/42 MAPK activation in rat vascular smooth muscle cells. $\mathrm{Br} \mathrm{J}$ Pharmacol 136: 613-619, 2002

7. Zhang J, Zhao F, Yu X, Lu X and Zheng G: MicroRNA-155 modulates the proliferation of vascular smooth muscle cells by targeting endothelial nitric oxide synthase. Int J Mol Med 35: $1708-1714,2015$

8. Delbosc S, Glorian M, Le Port AS, Bereziat G, Andreani M and Limon I: The benefit of docosahexanoic acid on the migration of vascular smooth muscle cells is partially dependent on Notch regulation of MMP-2/-9. Am J Pathol 172: 1430-1440, 2008.

9. Terano T, Tanaka T, Tamura Y, Kitagawa M, Higashi H, Saito Y and Hirai A: Eicosapentaenoic acid and docosahexaenoic acid inhibit vascular smooth muscle cell proliferation by inhibiting phosphorylation of Cdk2-cyclinE complex. Biochem Biophys Res Commun 254: 502-506, 1999.

10. Newell M, Baker K, Postovit LM and Field CJ: A critical review on the effect of docosahexaenoic acid (DHA) on cancer cell cycle progression. Int J Mol Sci 18: 1784, 2017.

11. Rani K and Aung NY: Docosahexaenoic acid inhibits vascular smooth muscle cell proliferation induced by glucose variability. Open Biochem J 11: 56-65, 2017.

12. Oono K, Takahashi K, Sukehara S, Kurosawa H, Matsumura T, Taniguchi S and Ohta S: Inhibition of PC3 human prostate cancer cell proliferation, invasion and migration by eicosapentaenoic acid and docosahexaenoic acid. Mol Clin Oncol 7: 217-220, 2017

13. Geng L, Zhou W, Liu B, Wang X and Chen B: DHA induces apoptosis of human malignant breast cancer tissues by the TLR-4/PPAR- $\alpha$ pathways. Oncol Lett 15: 2967-2977, 2018.

14. Sun Y, Wang K, Ye P, Wu J, Ren L, Zhang A, Huang X, Deng P, Wu C, Yue Z, et al: MicroRNA-155 promotes the directional migration of resident smooth muscle progenitor cells by regulating monocyte chemoattractant protein 1 in transplant arteriosclerosis. Arterioscler Thromb Vasc Biol 36: 1230-1239, 2016.

15. Li X, Kong D, Chen H, Liu S, Hu H, Wu T, Wang J, Chen W, Ning Y, Li Y and Lu Z: miR-155 acts as an anti-inflammatory factor in atherosclerosis-associated foam cell formation by repressing calcium-regulated heat stable protein 1 . Sci Rep 6 : 21789, 2016

16. Jin C, Cheng L, Lu X, Xie T, Wu H and Wu N: Elevated expression of miR-155 is associated with the differentiation of CD8+ T cells in patients with HIV-1. Mol Med Rep 16: 1584-1589, 2017.

17. Pacurari $M$ and Tchounwou PB: Role of MicroRNAs in renin-angiotensin-aldosterone system-mediated cardiovascular inflammation and remodeling. Int J Inflam 2015: 101527, 2015.

18. Izzard L, Dlugolenski D, Xia Y, McMahon M, Middleton D, Tripp RA and Stambas J: Enhanced immunogenicity following miR-155 incorporation into the influenza a virus genome. Virus Res 235: 115-120, 2017.

19. Heymans S, Corsten MF, Verhesen W, Carai P, van Leeuwen RE, Custers K, Peters T, Hazebroek M, Stöger L, Wijnands E, et al: Macrophage microRNA-155 promotes cardiac hypertrophy and failure. Circulation 128: 1420-1432, 2013.

20. Bhattacharya S, Chalk AM, Ng AJ, Martin TJ, Zannettino AC, Purton LE, Lu J, Baker EK and Walkley CR: Increased miR-155-5p and reduced miR-148a-3p contribute to the suppression of osteosarcoma cell death. Oncogene 35: 5282-5294, 2016

21. Ji Y, Wrzesinski C, Yu Z, Hu J, Gautam S, Hawk NV, Telford WG, Palmer DC, Franco Z, Sukumar M, et al: miR-155 augments CD8+ T-cell antitumor activity in lymphoreplete hosts by enhancing responsiveness to homeostatic gammac cytokines. Proc Natl Acad Sci USA 112: 476-481, 2015.

22. Kim S, Lee E, Jung J, Lee JW, Kim HJ, Kim J, Yoo HJ, Lee HJ, Chae SY, Jeon SM, et al: microRNA-155 positively regulates glucose metabolism via PIK3R1-FOXO3a-cMYC axis in breast cancer. Oncogene 37: 2982-2991, 2018.
23. Yang LX, Liu G, Zhu GF, Liu H, Guo RW, Qi F and Zou JH: MicroRNA-155 inhibits angiotensin II-induced vascular smooth muscle cell proliferation. J Renin Angiotensin Aldosterone Syst 15: 109-116, 2014

24. Zhang G, Zhong L, Luo $\mathrm{H}$ and Wang S: MicroRNA-155-3p promotes breast cancer progression through down-regulating CADM1. Onco Targets Ther 12: 7993-8002, 2019.

25. Livak KJ and Schmittgen TD: Analysis of relative gene expression data using real-time quantitative PCR and the 2(-Delta Delta C(T)) method. Methods 25: 402-408, 2001.

26. Wang CR, Zhu HF and Zhu Y: Knockout of microRNA-155 ameliorates the Th17/Th9 immune response and promotes wound healing. Curr Med Sci 39: 954-964, 2019.

27. Knolle MD, Chin SB, Rana BMJ, Englezakis A, Nakagawa R, Fallon PG, Git A and McKenzie ANJ: MicroRNA-155 protects group 2 innate lymphoid cells from apoptosis to promote type-2 immunity. Front Immunol 9: 2232, 2018.

28. Saleh M, Friedl A, Srivastava M, Soliman H, Secombes CJ and El-Matbouli M: STAT3/SOCS3 axis contributes to the outcome of salmonid whirling disease. PLoS One 15: e0234479, 2020

29. Oono K, Ohtake K, Watanabe C, Shiba S, Sekiya T and Kasono K: Contribution of Pyk2 pathway and reactive oxygen species (ROS) to the anti-cancer effects of eicosapentaenoic acid (EPA) in PC3 prostate cancer cells. Lipids Health Dis 19: 15, 2020.

30. Watanabe Y and Tatsuno I: Prevention of cardiovascular events with omega-3 polyunsaturated fatty acids and the mechanism involved. J Atheroscler Thromb 27: 183-198, 2020.

31. Farina FM, Hall IF, Serio S, Zani S, Climent M, Salvarani N, Carullo P, Civilini E, Condorelli G, Elia L and Quintavalle M: miR-128-3p is a novel regulator of vascular smooth muscle cell phenotypic switch and vascular diseases. Circ Res 126: e120-e135, 2020.

32. Nakagawa I, Park HS, Yokoyama S, Wada T, Yamada S, Motoyama Y, Kichikawa K and Nakase H: Pretreatment with and ongoing use of omega-3 fatty acid ethyl esters reduce the slow-flow phenomenon and prevent in-stent restenosis in patients undergoing carotid artery stenting. J Vasc Surg 66: 122-129, 2017.

33. Ding X, Ge L, Yan A, Ding Y, Tao J, Liu Q and Qiao C: Docosahexaenoic acid serving as sensitizing agents and gefitinib resistance revertants in EGFR targeting treatment. Onco Targets Ther 12: 10547-10558, 2019.

34. Song TJ, Chang Y, Shin MJ, Heo JH and Kim YJ: Low levels of plasma omega 3-polyunsaturated fatty acids are associated with cerebral small vessel diseases in acute ischemic stroke patients. Nutr Res 35: 368-374, 2015.

35. Morin C, Rousseau E, Blier PU and Fortin S: Effect of docosahexaenoic acid monoacylglyceride on systemic hypertension and cardiovascular dysfunction. Am J Physiol Heart Circ Physiol 309: H93-H102, 2015.

36. Yee D, Shah KM, Coles MC, Sharp TV and Lagos D: MicroRNA-155 induction via TNF- $\alpha$ and IFN- $\gamma$ suppresses expression of programmed death ligand-1 (PD-L1) in human primary cells. J Biol Chem 292: 20683-20693, 2017.

37. Xu L, Leng H, Shi X, Ji J, Fu J and Leng H: miR-155 promotes cell proliferation and inhibits apoptosis by PTEN signaling pathway in the psoriasis. Biomed Pharmacother 90: 524-530, 2017.

38. Seok HY, Chen J, Kataoka M, Huang ZP, Ding J, Yan J, Hu X and Wang DZ: Loss of MicroRNA-155 protects the heart from pathological cardiac hypertrophy. Circ Res 114: 1585-1595, 2014.

39. Wang J, Yu F, Jia X, Iwanowycz S, Wang Y, Huang S, Ai W and Fan D: MicroRNA-155 deficiency enhances the recruitment and functions of myeloid-derived suppressor cells in tumor microenvironment and promotes solid tumor growth. Int $\mathbf{J}$ Cancer 136: E602-E613, 2015.

40. Wan YC, Li T, Han YD, Zhang HY, Lin H and Zhang B: MicroRNA-155 enhances the activation of Wnt/ $\beta$-catenin signaling in colorectal carcinoma by suppressing HMG-box transcription factor 1. Mol Med Rep 13: 2221-2228, 2016.

41. Tu YX, Wang SB, Fu LQ, Li SS, Guo QP, Wu Y, Mou XZ and Tong XM: Ovatodiolide targets chronic myeloid leukemia stem cells by epigenetically upregulating hsa-miR-155, suppressing the BCR-ABL fusion gene and dysregulating the PI3K/AKT/mTOR pathway. Oncotarget 9: 3267-3277, 2017.

42. Zhang L, Wang W, Li X, He S, Yao J, Wang X, Zhang D and Sun X: MicroRNA-155 promotes tumor growth of human hepatocellular carcinoma by targeting ARID2. Int J Oncol 48: 2425-2434, 2016 
43. Liu D, Han P, Gao C, Gao W, Yao X and Liu S: microRNA-155 modulates hepatic stellate cell proliferation, apoptosis, and cell cycle progression in rats with alcoholic hepatitis via the MAPK signaling pathway through targeting SOCS1. Front Pharmacol 11: $270,2020$.

44. Chen L, Ming X, Li W, Bi M, Yan B, Wang X, Yang P and Yang B: The microRNA-155 mediates hepatitis B virus replication by reinforcing SOCS1 signalling-induced autophagy. Cell Biochem Funct 38: 436-442, 2020.

45. Ye J, Guo R, Shi Y, Qi F, Guo C and Yang L: miR-155 regulated inflammation response by the SOCS1-STAT3-PDCD4 axis in Atherogenesis. Mediators Inflamm 2016: 8060182, 2016.
46. Yang Y, Yang L, Liang X and Zhu G: MicroRNA-155 promotes atherosclerosis inflammation via targeting SOCS1. Cell Physiol Biochem 36: 1371-1381, 2015.

47. Bouamar H, Jiang D, Wang L, Lin AP, Ortega M and Aguiar RC: MicroRNA 155 control of p53 activity is context dependent and mediated by Aicda and Socs1. Mol Cell Biol 35: 1329-1340, 2015.

(i) $\odot$ This work is licensed under a Creative Commons Attribution-NonCommercial-NoDerivatives 4.0 International (CC BY-NC-ND 4.0) License. 